\title{
SCIENTIFIC CONTENTS OF THE HIPPARCOS INPUT CATALOGUE ${ }^{1}$
}

\author{
C. TURON, A.E. GOMEZ, D. MORIN \\ DASGAL/URA 335 du CNRS, Observatoire de Paris-Meudon \\ 92195 Meudon Cedex, FRANCE \\ e-mail: turon@obspm.fr
}

\section{Introduction}

The Hipparcos Input Catalogue -hereafter called HIC- (Turon et al. 1992a, Turon et al. 1994) contains the observing programme of the Hipparcos mission: 118000 preselected stars, 48 minor planets and three satellites of major planets. These objects were selected on the grounds of more than 200 scientific programmes proposed by the world-wide astronomical community, and dealing with a large variety of astronomical and astrophysical topics. It contains the most up-to-date, comprehensive and homogeneous data and information related to these programme stars, collected during the years 1981-1990 by the INCA Consortium (Hipparcos INput CAtalogue consortium).

The accuracy of the astrometric and photometric parameters obtained from the analysis of the first 30 months of Hipparcos data already provides a check the various sources of ground-based data contained in the Hipparcos Input Catalogue. It also provides a statistical estimation of what can be expected for the various types of scientific programmes proposed for observation by Hipparcos, in terms of accuracy of the astrometric parameters with respect to spectral types, luminosity classes, types of objects, etc.

\footnotetext{
${ }^{1}$ Based on observations made with the ESA Hipparcos satellite, and on work performed within the INCA, FAST and NDAC Consortia. 
2. The quality of the astrometric and photometric data in the Hipparcos Input Catalogue

The data collected in the HIC were obtained from extensive cooperative programmes of compilation and new observations or measurements performed by the INCA Consortium during the years 1981-1990. A complete description of the data included in the HIC can be found in the introduction to the printed, tape and CD-ROM versions of the Hipparcos Input Catalogue (Turon et al. 1992a, Turon et al. 1992b, Turon et al. 1994) and in Perryman and Turon 1989.

\subsection{POSITIONS}

The positions available from the 30 -month combined sphere solution (H30) are of an unprecedent accuracy as compared with ground-based positions: median rms of 1.1 and 0.9 mas for ecliptic longitude and latitude respectively. These values are about $1 / 20$ of the rms claimed by the most accurate ground-based catalogues, and confirm that these preliminary Hipparcos results are perfectly suitable for our present purpose. The distribution of differences between $\mathrm{H} 30$ and IC8 (version of HIC used for satellite observations) is illustrated in Table 1. Only single entries were considered for the comparisons by source catalogue.

In order to characterise the scatter of these differences, a width based on distribution percentiles is used as dispersion estimate instead of a rms scatter, which is too sensitive to heavy tail distributions and outliers.

\subsection{PROPER MOTIONS}

The provisional proper motions available from the 30 -month combined solution show median rms of 1.5 and $1.3 \mathrm{mas} / \mathrm{yr}$ for the proper motion in longitude and in latitude respectively. These values will be improved in the final data reduction, including the 37 months of Hipparcos data. Therefore, the results shown here are only tentative. However, for all source catalogues with the exception of the FK5, these preliminary results should be significant. The distribution of differences between H30 and IC8 is illustrated in Table 2. 
TABLE 1. Median and width ( $\operatorname{arcsec})$ of distribution of differences between $\mathrm{H} 30$ and IC8 in $\alpha \cos \delta$ and $\delta$ (equinox J2000.0, epoch J1990.0)

\begin{tabular}{|c|c|c|c|c|c|}
\hline & \multirow{2}{*}{$\begin{array}{l}\mathrm{Nb} \text { of } \\
\text { stars }\end{array}$} & \multicolumn{2}{|c|}{$\alpha \cos \delta$} & \multicolumn{2}{|c|}{$\delta$} \\
\hline & & median & width & median & width \\
\hline All stars & 107495 & -0.020 & 0.275 & 0.070 & 0.285 \\
\hline Single entries & 104045 & -0.020 & 0.275 & 0.070 & 0.280 \\
\hline Joint entries & 3450 & -0.060 & 0.330 & 0.080 & 0.310 \\
\hline FK5 & 1940 & 0.005 & 0.080 & 0.040 & 0.095 \\
\hline Meridian & 6283 & 0.000 & 0.108 & 0.040 & 0.140 \\
\hline IRS * & 12629 & -0.030 & 0.185 & 0.080 & 0.200 \\
\hline SSSC & 3851 & -0.270 & 0.255 & 0.170 & 0.248 \\
\hline $\mathrm{PPM} *$ & 41448 & 0.030 & 0.270 & 0.040 & 0.285 \\
\hline $\mathrm{CPC} 2 *$ & 9585 & -0.240 & 0.320 & 0.160 & 0.295 \\
\hline Plate meas. & 12199 & -0.030 & 0.355 & 0.080 & 0.405 \\
\hline
\end{tabular}

* provisional versions

TABLE 2. Median and width (arcsec) of distribution of differences between $\mathrm{H} 30$ and IC8 in $\mu_{\alpha} \cos \delta$ and $\mu_{\delta}$

\begin{tabular}{lrrrrr}
\hline & \multicolumn{2}{c}{ Nb of } & \multicolumn{2}{c}{$\mu_{\alpha} \cos \delta$} & \multicolumn{2}{c}{$\mu_{\delta}$} \\
& stars & median & width & median & width \\
\hline single entries & 90619 & 0.000 & 0.007 & 0.001 & 0.008 \\
& & & & & \\
FK5 & 1940 & 0.000 & 0.003 & 0.000 & 0.003 \\
Meridian & 5273 & 0.000 & 0.002 & 0.000 & 0.005 \\
IRS ${ }^{*}$ & 7558 & 0.000 & 0.006 & 0.001 & 0.006 \\
SSSC & 3440 & 0.000 & 0.011 & 0.007 & 0.009 \\
PPM $^{*}$ & 41451 & 0.001 & 0.006 & 0.000 & 0.007 \\
CPC2 & 5784 & -0.005 & 0.006 & 0.003 & 0.005 \\
SAO & 11605 & 0.000 & 0.017 & 0.000 & 0.017 \\
& & & & & \\
\hline
\end{tabular}

* provisional versions 


\subsection{PHOTOMETRY}

Within the frame of the Hipparcos Input Catalogue preparation, Hp magnitudes (magnitudes in the wide bandpass of the Hipparcos photometric system, Grenon et al. 1992), had to be predicted from ground-based multicolour photometry, in order to optimise the observing time allocation, and to check the star observability. In addition, photoelectric photometry was collected to produce high accuracy standards for the on-orbit calibration of the detection chain. The accuracy of Hipparcos photometric measurements is at the level of a few millimagnitudes. The comparison of these measurements with those included in the HIC is illustrated in Table 3.

TABLE 3. Median and width (magnitudes) of distribution of differences between $\mathrm{H} 30$ and $\mathrm{HIC}$ in $\mathrm{Hp}$ magnitudes

\begin{tabular}{lccrc}
\hline & Number of stars & median & width \\
& 117610 & $\mathrm{~J}$ & 0.000 & 0.098 \\
all stars & 44483 & 0.000 & 0.016 \\
photoelectric B and V & 13298 & -0.031 & 0.064 \\
photoelectric V & 45273 & 0.022 & 0.240 \\
heterogeneous $\mathrm{V}^{*}$ & & & & \\
\hline
\end{tabular}

* colour derived from spectral type and extinction model (Arenou et al. 1992)

\subsection{CONCLUSION}

The astrometric and photometric data included in the Hipparcos Input Catalogue are largely within the original specifications of ESA: \pm 0.3 arcsec on 1990 positions (to be compared with a specification of $1.5 \operatorname{arcsec}$ ); \pm $0.25 \mathrm{mag}$ on $\mathrm{Hp}$ magnitudes and $\pm 0.02 \mathrm{mag}$ for more than a third of the catalogue (to be compared with a specification of $0.5 \mathrm{mag}$ ). 


\section{Expectations with respect to scientific objectives}

The 118000 stars were selected on the grounds of more than 200 scientific programmes proposed by the world-wide astronomical community, and dealing with a large variety of astronomical and astrophysical topics: fundamental astronomy (reference systems; dynamics of the stellar system; astrometry); stellar physics (stellar luminosities; fine structure of the HR diagram; tests of the physics involved in internal stellar structure models; stellar evolution; determination of stellar ages, masses, and diameters; etc); galactic physics (stellar populations; chemical, kinematical and dynamical evolution of our Galaxy; galactic rotation and potential; stellar orbits; etc); cosmic distance scale (new calibrations of photometric and spectroscopic parallaxes, of period-luminosity relations; detailed study of the effects of chemical abundances - metallicity, Helium, Oxygen, etc- on the location of the main sequence or giant branch).

Details on the iterative process which led to selection of the final observing programme are given in Turon et al. 1992c. The results obtained from the analysis of the first 30 months of Hipparcos data already give a very good confidence in the possibilities expected from the final Hipparcos Catalogue:

- with respect to fundamental astrometry: the Hipparcos reference frame will be characterised by an accuracy of 1.5 mas for positions at epoch 1991.25 and of $1.5 \mathrm{mas} / \mathrm{yr}$ for proper motions; a link to an extragalactic system with an accuracy of 0.5 mas on the orientation $R, 0.3$ mas $/ y r$ on the rotation R'; a stellar density of 3 stars per square degree.

- with respect to stellar physics: the present accuracy already allows a direct determination of luminosities in the range of $M_{V}-4$ to $14 \mathrm{mag}$; about 200 stars with a relative accuracy of the trigonometric parallax better than 1\% (a few giants and subgiants, from F2 to K2; dwarfs from A0 to M5; one white dwarf); 3300 stars with a relative accuracy of the trigonometric parallax better than $5 \%$ (a few supergiants; about 200 giants, from B7 to M4; about 300 subgiants, from B2 to K4; about 2000 dwarfs, from B3 to M8; 10 white dwarfs, 50 orbital systems; a few pulsating stars; etc); 13000 stars with a relative accuracy of the trigonometric parallax better than $10 \%$ (up to B0 stars on the main sequence; about 1000 giants, from B5 to M5).

- with respect to galactic physics and cosmic distance scale determinations: 13000 stars with a relative accuracy of the trigonometric parallax better than 10\%; 40000 stars with a relative accuracy of the trigonometric parallax better than 20\%; 36000 stars with a relative 
accuracy of the proper motion better than $5 \% ; 82000$ stars with a relative accuracy of the proper motion better than $20 \%$; direct distance determination of about 20 galactic open clusters of various ages and metallicities; direct distance determination of a few pulsating stars, supergiants stars and bright giants, planetary nebulae, novae, etc; discovery of new variable stars.

\section{Conclusions}

The results obtained with only 30 months of data already allow to deal with all the categories of astrometric and astrophysical programmes submitted by the worldwide astronomical community in 1982 and the Hipparcos Consortia members in 1993. The analysis of the totality of the 37 month mission will still improve the accuracy of the astrometric parameters and the coherence of the whole solution. Moreover, the 'Hipparcos sphere' will be linked to extragalactic reference system, leading to an absolute determination of the proper motions. In parallel, a new reduction of the photometric data is being performed, using an improved set of standard stars and calibrations.

\section{References}

Arenou, F., Grenon M., Gómez, A.E. (1992) $A \& A, \mathbf{2 5 8}, 104$

Grenon M., Mermilliod, J.C., Mermilliod, M. (1992) $A \& A$, 258, 88

Perryman M.A.C., Turon C. (eds), 1989, ESA SP-1111, Vol. II

Turon, C. et al. (1992a) The Hipparcos Input Catalogue, ESA SP-1136.

Turon C. et al. (1992b), Bull. Inform. CDS, 41, 9

Turon, C., Gómez, A., Crifo, F., Crézé, M., Perryman, M.A.C., Morin, D., Arenou, F., Nicolet, B., Chareton, M., Egret, D. (1992c), A\&A 258, 74

Turon, C., Morin, D., Arenou, F., Perryman M.A.C. and Priou D. (1994) CD-ROM version of the Hipparcos Input Catalogue, Turon, C. ed 